\section{Bradley J. Close \\ Peter J.Strouse}

Received: 25 October 1999

Accepted: 6 June 2000

B. J. Close ( $)$ P. J. Strouse

Section of Pediatric Radiology,

C.S. Mott Children's Hospital,

University of Michigan Health System,

1500 East Medical Center Drive,

Ann Arbor, MI 48109-0252, USA

\title{
MR of physeal fractures of the adolescent knee
}

\begin{abstract}
The aim of this study was to assess physeal fractures of the pediatric knee identified by MR imaging and to describe the MR findings of such fractures. The authors reviewed 315 consecutive pediatric knee MR examinations done to assess for traumatic injury. The MR images were reviewed for evidence of physeal fracture. Fractures were classified by the Salter-Harris system, and associated findings and injuries were noted. Plain radiographs and medical records were reviewed. Seven distal femoral physeal fractures (Salter II, $n=6$; Salter III, $n=1$ ) and two proximal tibia physeal fractures (Salter III, $n=1$; complex Salter IV, $n=1$ ) were identified. Magnetic resonance demonstrated widening of a portion of the physis with visualization of a
\end{abstract}

metaphyseal/epiphyseal fracture line. Periosteal elevation was observed in six cases. Four patients had associated ligamentous or meniscal injuries. Plain radiographs were available for review in eight patients. Bone abnormalities suggesting fracture were evident in six of eight patients; however, the fracture was fully delineated in only one patient. The diagnosis or confirmation of fracture by MR changed clinical management in seven of eight patients in whom follow-up was available. Physeal fractures of the pediatric knee are occasionally diagnosed by MR. Magnetic resonance provides improved delineation of non-displaced physeal fractures of the knee, while simultaneously allowing for evaluation of soft tissue structures.

\section{Introduction}

Several authors have described the use of MR imaging in evaluating various types of fractures. Magnetic resonance has been proven to be valuable both in identifying occult fractures of the adult knee and in evaluating Salter fractures of various bones in the young child [1-8]. Magnetic resonance has shown prowess in assessing the post-traumatic knee for internal derangement, both in adult and pediatric patients $[9,10]$. Descriptions of MR demonstration of physeal injuries in the adolescent knee are sparse. In this study, we retrospectively evaluated pediatric patients undergoing knee MR for evidence of physeal fracture. We studied these cases to determine the MR findings of physeal fracture at the knee, whether the fractures were radiographically evident, and whether MR diagnosis or confirmation of fracture changed patient management.

\section{Materials and methods}

Three hundred forty-five knee MR studies performed in pediatric patients aged 18 years or less from January 1994 to March 1998 were reviewed. We excluded 30 examinations in which MR was performed for reasons other than assessing traumatic injury (mass, $n=10$, known osteochondritis dissecans, $n=10$, avascular necrosis, $n=3$, miscellaneous other indications, $n=7$ ). This left 315 knee MR examinations performed on 289 patients and 308 knees. In 14 patients, both knees were imaged on the same date. Five patients had both knees examined, but on different dates. 

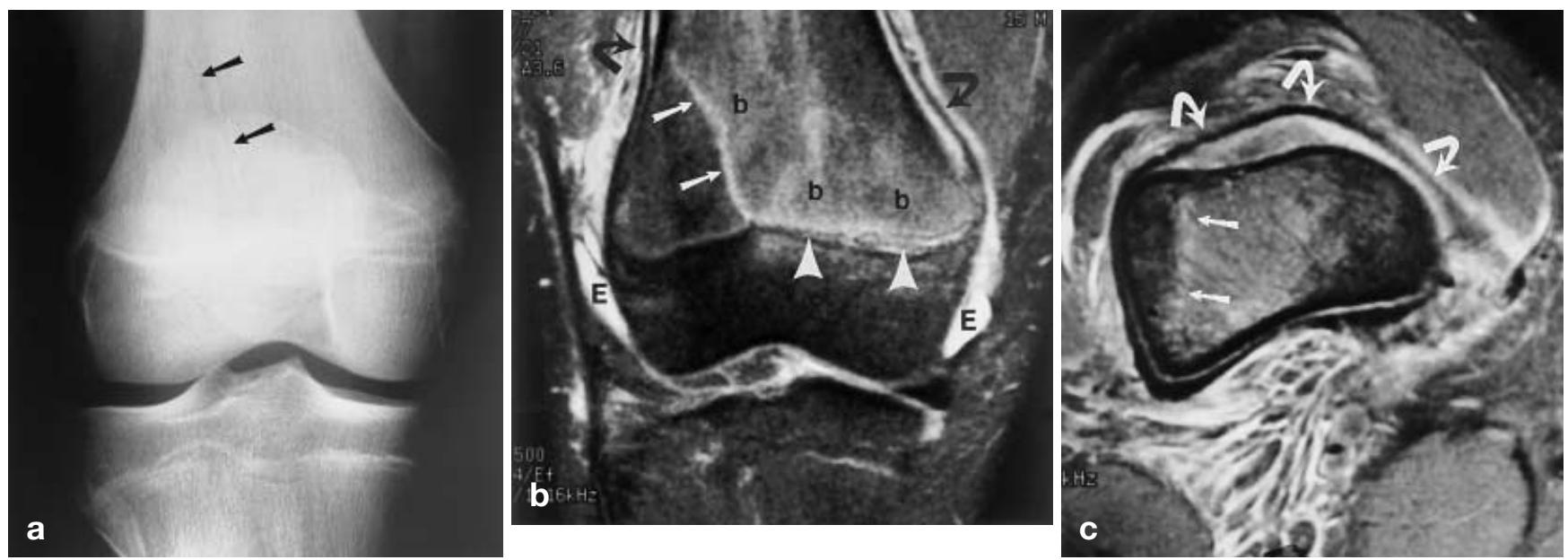

Fig. 1a-c A 15-year-old boy with Salter II fracture of the distal femur. a Plain radiograph shows a faint metaphyseal fracture line (arrows) that was noted only in retrospect. b Coronal proton-density with fat-saturation MR image shows the same metaphyseal fracture line (straight arrows), widening of the medial physis (arrow heads), metaphyseal bone marrow edema and periosteal elevation (curved arrows; $E$ effusion). c Axial proton density with fat-saturation MR image better demonstrates periosteal elevation (curved arrows). A band of high signal represents the metaphyseal fracture line, not well seen in the axial plane (straight arrows)

Seven patients had two separate examinations of the same knee. One hundred ninety studies were performed on 175 boys and 125 studies on 114 girls. For the group as a whole, the age range was 3-18 years with a mean age of 14.4 years and median age of 15 years. For subgroups of boys and girls, the age range was also $3-18$ years and the median age 15 years; however, the mean age for girls was 14.0 years and for boys was 14.6 years.

All knee MR studies were performed under a standard protocol on a 1.5-T magnet (Signa, GE Medical Systems, Milwaukee, Wis.). An extremity coil was used, with the knee placed in $15^{\circ}$ of external rotation to profile the anterior cruciate ligament in the sagittal plane better. Our standard pediatric knee MR protocol was modified over the period of study. Older examinations were obtained with the following protocol:

1. Localizer: axial T1-weighted fast spin echo (FSE; TR $500 \mathrm{~ms}$, TE $17 \mathrm{~ms}, 10 \mathrm{~mm}$ thick skipping $2 \mathrm{~mm}$ )

2. Axial T1-weighted SE (TR $600 \mathrm{~ms}$, minimum TE, $4 \mathrm{~mm}$ thick skipping $0.5 \mathrm{~mm}$ )

3. Sagittal T1-weighted SE (TR $650 \mathrm{~ms}$, minimum TE, $3 \mathrm{~mm}$ thick skipping $1 \mathrm{~mm}$ )

4. Sagittal 3D gradient echo (GRE; TR $31 \mathrm{~ms}$, TE $15 \mathrm{~ms}$, flip angle $30^{\circ}, 1.5 \mathrm{~mm}$ thick with no skip

5. Coronal proton density/T2-weighted SE (TR $4500 \mathrm{~ms}$, TE 17 and $85 \mathrm{~ms}, 4 \mathrm{~mm}$ thick skipping $0.5 \mathrm{~mm}$ )

Our current protocol is:

1. Localizer: axial T1-weighted FSE (same parameters)

2. Axial proton density with fat saturation FSE (TR $4000 \mathrm{~ms}$, TE $17 \mathrm{~ms}, 4 \mathrm{~mm}$ thick skipping $0.5 \mathrm{~mm}$ )

3. Sagittal proton density SE (TR $1200 \mathrm{~ms}$, TE $12 \mathrm{~ms}, 3 \mathrm{~mm}$ thick skipping $1 \mathrm{~mm}$ )
4. Sagittal 3D GRE (same parameters)

5. Coronal proton density FSE (TR $4500 \mathrm{~ms}$, TE $17 \mathrm{~ms}, 4 \mathrm{~mm}$ thick skipping $0.5 \mathrm{~mm}$ )

6. Coronal proton density with fat saturation FSE (TR $4500 \mathrm{~ms}$, minimum TE, $4 \mathrm{~mm}$ thick skipping $0.5 \mathrm{~mm}$ )

Magnetic resonance images were reviewed to identify all patients with fractures of the distal femur or proximal tibia involving the physis. Fractures were identified as a well-defined linear signal abnormality within the metaphysis and/or epiphysis, low signal on T1-weighted images, and high signal on T2-weighted images. Physeal portions of fractures were identified by localized physeal widening and increased signal on T2-weighted images. The fractures were categorized by the Salter-Harris system [11]. Associated injuries and abnormalities were noted. Although not required, most of our patients undergo routine knee radiography prior to MR, usually at the time of initial clinical evaluation. For patients seeing our orthopedic surgeons, anteroposterior, lateral, notch, and patellar views are routinely obtained. Oblique and cross-table lateral radiographs are not routinely obtained. Plain radiographs of each patient with physeal fractures seen by MR were reviewed for evidence of fracture. Medical records were reviewed to identify mechanisms of injury and to determine if MR diagnosis or confirmation of fracture changed clinical mnagement.

\section{Results}

Nine patients ( 7 male and 2 female) were determined to have a physeal fracture at the knee by MR. The overall incidence of physeal fracture was $2.9 \%$ (9 of 315 patients). The average age of the seven males was 15.1 years (age range $14-17$ years), with the two females being 11 and 12 years old. The average interval from time of injury was 8.6 days (range 4-20 days).

Six fractures were classified as Salter II, two were classified as Salter III, and one was classified as a complex Salter IV fracture. All six Salter II fractures involved the distal femur, with five of the six cases demonstrating the fracture line to extend through the medial physis and lateral metaphysis (Figs. 1, 2). The other distal femoral Salter II fracture was through the 

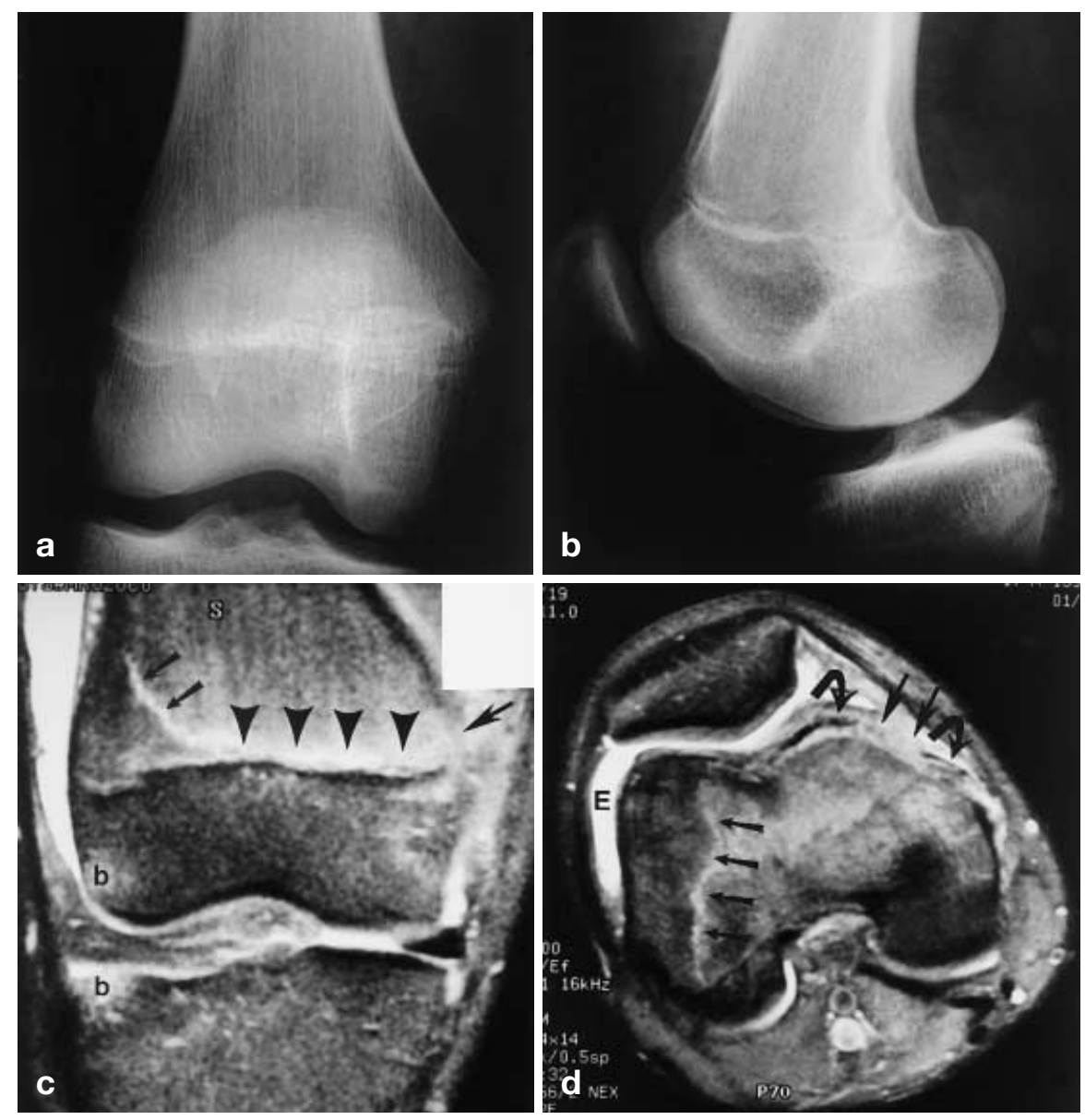

Fig. 2a-d A 17-year-old boy with Salter II fracture of the distal femur. a, b Anteroposterior and lateral radiographs of the right knee demonstrate no bony abnormality. Soft tissue planes in the distal thigh are indistinct. This was better appreciated by "hot lighting" these overexposed films. The suprapatellar pouch is not well defined; however, no fullness is evident to suggest effusion. c Coronal proton density with fat saturation MR image shows a metaphyseal fracture line laterally (small arrows). Increased signal is noted within the medial physis (arrowheads) with adjacent metaphyseal bone marrow edema. Small areas of bone marrow edema are also seen in the lateral aspect of the distal femoral and proximal tibial epiphyses $(b)$. Periosteal disruption is noted medially (large arrow). E joint effusion. d Axial proton density with fat saturation MR image obtained immediately above the distal femoral physis shows the metaphyseal fracture line (small arrows). High signal in the central and medial femur may represent bone marrow edema and/or physeal injury. The periosteum is disrupted (large arrows) and minimally elevated medially (curved arrows; $E$ joint effusion)

lateral physis and medial metaphysis. Of the two Salter III fractures, one occurred at the distal femur with extension of the fracture line through the medial physis (Fig. 3), whereas the other extended from the proximal tibial epiphysis through the medial physis. The remaining fracture involved the proximal tibial epiphysis,

metaphysis, and probably the intervening physis (Fig.4). Since the epiphyseal and metaphyseal fracture lines were not completely in plane and the extent of physeal fracture unclear, this fracture was classified as a complex Salter IV fracture.

The MR findings seen in association with these fractures included marrow edema, effusion, ligament injury, meniscal injury, and periosteal elevation. High signal on T2-weighted images, indicative of bone marrow edema, was seen in association with all of the fractures. In each of the patients with distal femur fractures, bone marrow edema was adjacent to fracture within the metaphysis and to a lesser extent within the epiphysis. Three of these patients had additional marrow edema of the lateral femoral condyle and one had proximal tibial epiphyseal marrow edema. Minimal lateral femoral condyle and moderate anterolateral tibial plateau marrow edema was seen with the tibial Salter III fracture. Minimal bone marrow edema was seen adjacent to fracture lines of the tibial Salter IV fracture. A suprapatellar knee effusion was noted in six of the nine patients on MR. Ligamentous and/or meniscal injuries were found in four patients, with two anterior cruciate ligament tears, one medial collateral ligament tear, and three me- 
Fig.3a-d A 14-year-old boy with Salter III fracture of the distal femur. a Outside plain radiograph shows widening of the medial physis (arrowheads) and irregularity within the intercondylar region (arrow). b Coronal proton-densityweighted MR shows a vertical epiphyseal fracture (arrows), widening of the medial physis (arrowheads), and periosteal elevation (curved arrow). Axial T2-weighted MR images demonstrate $\mathbf{c}$ the sagittal epiphyseal fracture line (arrows) and d medial periosteal elevation (curved arrows). Note sites of periosteal disruption (straight arrows). A moderately large joint effusion $(E)$ is present
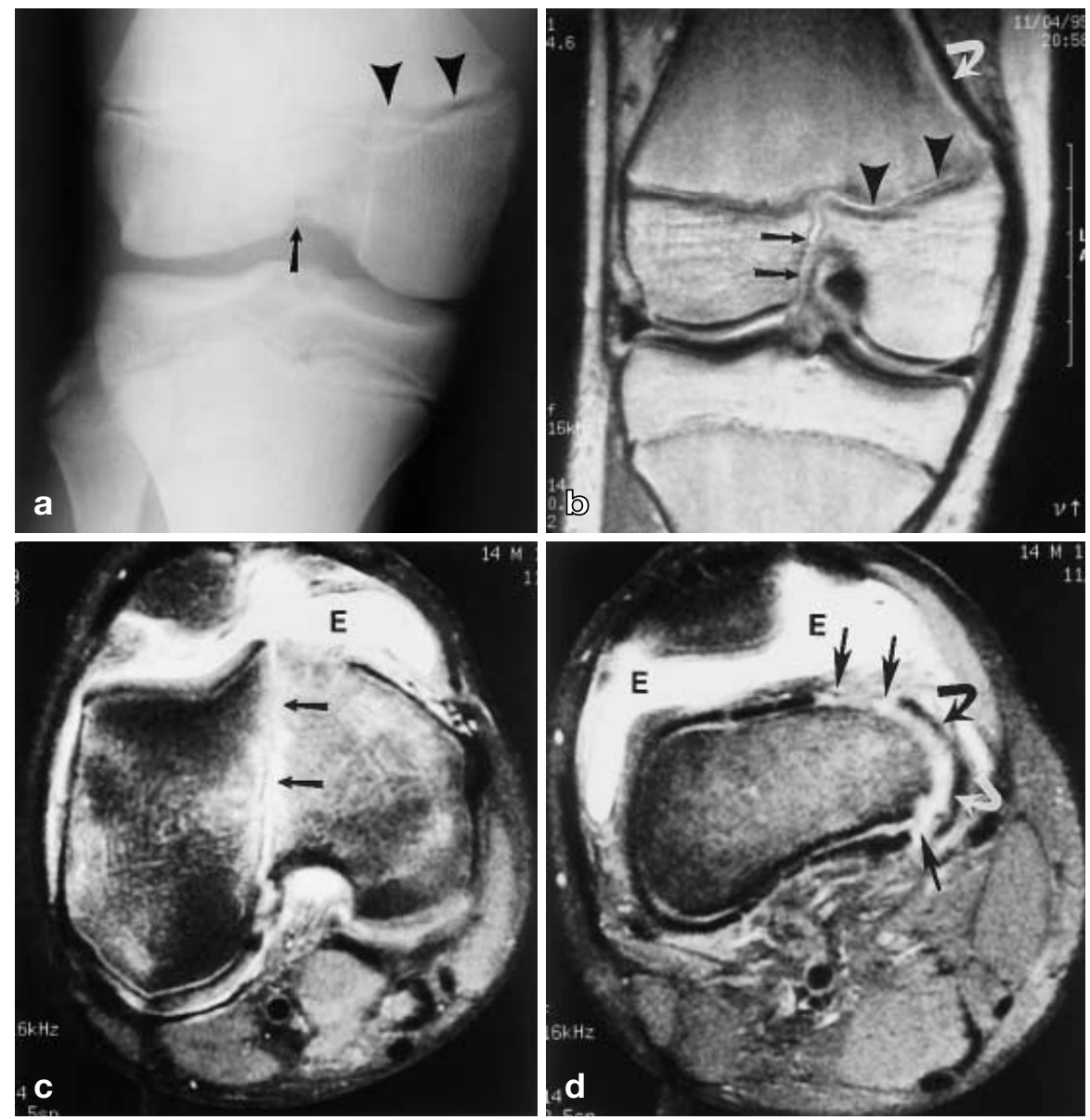

niscal injuries. The patient with the complex Salter IV fracture of the proximal tibia was noted to have an intact discoid lateral meniscus. Periosteal elevation was noted in six patients and periosteal disruption in four patients. In each instance the periosteum was elevated on the side of physeal fracture (i.e., fracture line through the medial physis was associated with periosteal elevation medially). In two cases periosteal elevation was also noted on the metaphyseal side of a Salter II fracture, one of which demonstrated diffuse anterior periosteal elevation as well. Associated periosteal disruption was seen on the physeal side of fracture in three patients and the metaphyseal side of fracture in one patient.

All nine patients had plain radiographs prior to MR, with radiographs of eight available for review. Bone abnormalities diagnostic or suggestive of fracture were present in six patients. In only one case, the Salter III fracture of the proximal tibia, was the full extent of fracture evident on the radiographs. Soft tissue swelling with partial obliteration of soft tissue planes was seen in five of eight patients, four of whom had a bone abnormality. Effusion within the suprapatellar pouch was seen in six of eight patients, five of whom had a bone abnormality.

Medical records from the emergency room or clinic prior to the MR were available for six patients. The interval from injury to MR varied up to 20 days. Clinical data is therefore limited and varied considerably from patient to patient. Excluding the patient in whom plain radiographs firmly diagnosed and fully delineated the fracture, no mention of fracture was provided within the clinical history on the MR requisition or on the patient questionnaire filled out at the time of the MR examination.

Injuries were incurred while playing football in three patients, and in one patient each playing soccer, playing hockey, running, skiing, and falling off of a bicycle. In one patient there was no history available on the mechanism of injury. Activities leading to injury did not vary considerably from activities causing ligamentous or meniscal injury in other patients. Descriptions of the injuries were inadequate to draw conclusions on mechanism of fracture. For instance, no consistent mechanism (i.e., valgus stress) was elicited for the five distal femoral Salter II fractures with lateral metaphyse- 

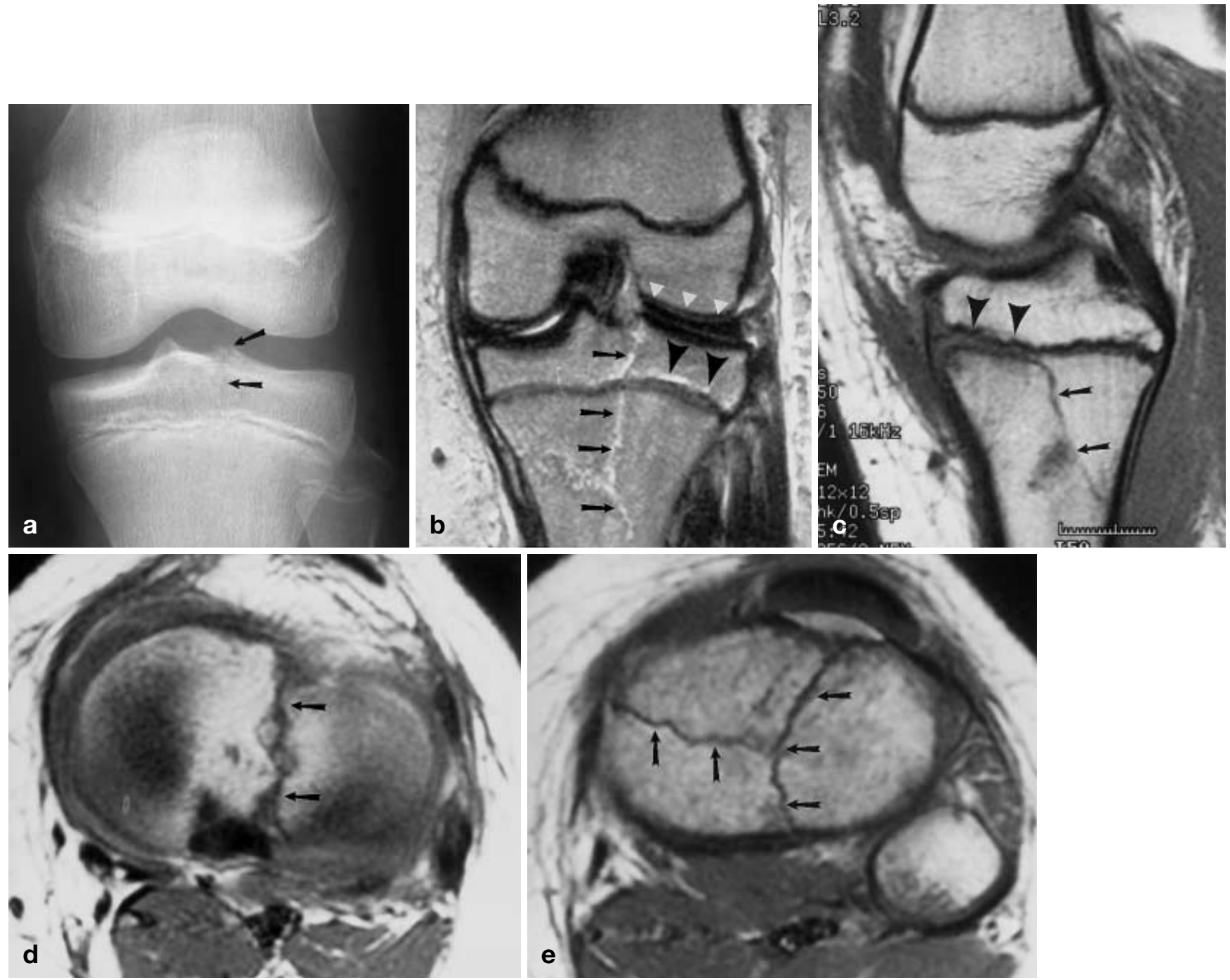

Fig. 4a-e An 11-year-old girl with complex physeal fracture. a Plain radiograph only shows irregularity of the tibial spine with a irregular vertical lucency extending inferiorly through the epiphysis (arrows). b Coronal T2-weighted MR image shows sagittal epiphyseal and metaphyseal fracture lines (arrows). Increased signal is noted adjacent to the lateral physis (black arrowheads). The lateral meniscus (white arrowheads) is discoid but intact. c Sagittal T1-weighted MR image shows a coronally oriented metaphyseal fracture line (arrows) and apparent widening of the anterior physis (arrowheads). Axial T1-weighted MR images show d the sagittal epiphyseal fracture line (arrows) and e the sagittal (horizontal arrows) and coronal metaphyseal fracture lines (vertical arrows). The extent of physeal fracture was unclear in this patient. The sagittal T1-weighted and GRE images showed apparent widening of the anteromedial physis in contiguity with the coronal metaphyseal fracture line; however; the coronal T2-weighted image only demonstrated increased signal along the lateral physis al/medial physeal planes of fracture. Follow-up clinical information was available for eight patients. Medical management was altered in seven of these eight patients based on the MR diagnosis or confirmation of fracture (cast, $n=4$; prolonged non-weight bearing, $n=2$; change to brace, $n=1)$.

\section{Discussion}

This study demonstrates to ability of MR to diagnose and delineate physeal fractures of the adolescent knee. The ability of MR to assess for occult fractures in the adult knee and the ability of MR to assess physeal fractures in the young child have both been established [1-8]. Magnetic resonance has been shown to modify the classification of physeal fractures significantly [1,2, 4]. No previous studies have specifically examined MR findings of physeal fracture in the adolescent knee. 
Two previous investigations studied the spectrum of injury evident in the injured pediatric knee on MR [9, 10]. Zobel et al. [9] mention three "nonosteochondral" fractures in a study of 104 pediatric patients, but do not characterize the fractures. King et al. [10] do not mention any nonosteochondral fractures at all in a study of 78 pediatric patients.

Physeal fractures are not uncommon. The large majority of physeal fractures in the adolescent knee are diagnosed by plain-film radiography, without the need for MR. The incidence of physeal fractures identified by MR in pediatric patients who undergo knee MR to evaluate traumatic injury is $2.9 \%$ at our institution based on this study. This number is higher than that inferred by prior studies of pediatric knee MR. Several potential explanations exist:

1. We may be diagnosing more physeal fractures by MR because of a failure to diagnose them by plain-film radiography.

2. Our clinical colleagues may be ordering the MR scans to delineate suspected fractures, although this has not been communicated to us.

3. Out patient population may be skewed to include a higher percentage of patients involved in competitive or contact sports, contributing to a higher fracture rate.

4. Patient selection bias contributes, as prospective identification of some of the fractures included in this study prompted the investigation.

5. These fractures may have been under-diagnosed in prior studies. Improved MR scanners and sequences better delineate the fractures.

Evaluation of a patient with knee trauma begins with a complete history and physical examination. Plain radiographs are the initial mode of imaging. Most fractures are grossly evident on physical examination or radiographs. Careful assessment of the radiographs should not be limited to evaluation of the bone, but should include assessment of periarticular soft tissue planes and of joint effusion. Equivocal findings may warrant the addition of oblique and cross-table lateral radiographs. Non-displaced physeal fractures may be less apparent on physical examination and subtle on radiography. In these patients MR can be performed to elucidate a cause for pain or to confirm and delineate a suspected fracture. Systematic assessment of the MR images should always include a search for findings of physeal fracture, i. e., physeal widening, epiphyseal or metaphyseal fracture lines, bone marrow edema, and periosteal elevation or disruption. Diagnosis of a fracture does not preclude the presence of an associated ligamentous or meniscal injury [12].

Periosteal elevation was seen in association with a majority of the fractures in our series. According to the orthopedic literature, although separated from the metaphyseal cortex, periosteum along the compression side (side of the metaphyseal fracture in a Salter II fracture) usually is intact, whereas that on the distraction side (side of the physeal fracture in a Salter II fracture) is frequently torn. A greater degree of elevation or displacement from the underlying cortex is therefore expected on the distraction side [13]. This was true of our patients with Salter II fractures. The ancillary MR finding of periosteal elevation offers confirmatory evidence of fracture or may prompt the search for a subtle fracture not previously noted.

A limitation of this study is the acceptance of MR as a gold standard for the presence of fracture. We believe MR to be accurate. The ability of MR to diagnose fracture is accepted [1-8]. The findings of physeal widening and metaphyseal or epiphyseal fracture lines are difficult to dispute. Subtle plain-film findings are supportive. On limited plain-film follow-ups the metaphyseal fractures have shown signs of healing.

As seen in our patients, diagnosis or confirmation of a fracture by MR will change management in a majority of patients, namely, by implementation of immobilization and non-weight-bearing. These measures serve to relieve symptoms, promote healing, and diminish the risk of complications (displacement, non-union, growth arrest).

In conclusion, we present the MR findings of nine patients with physeal fractures of the knee, diagnosed or confirmed by MR. Magnetic resonance better delineated the fractures than plain radiographs. Evaluation of an adolescent's knee MR should include a specific search for findings of physeal fracture. Magnetic resonance can be used to investigate questionable plainfilm bone abnormalities, offering the benefit of simultaneously evaluating for soft tissue injury.

\section{References}

1. Smith BG, Rand F, Jaramillo D, et al (1994) Early MR imaging of lower-extremity physeal fracture-separation: a preliminary report. J Pediatr Orthop 14: $526-533$
2. Beltran J, Rosenberg ZS, Kawelblum M, et al (1994) Pediatric elbow fractures: MR evaluation. Skeletal Radiol 23: $277-281$

3. Jaramillo D, Hoffer FA, Shapiro F, et al (1990) MR imaging of fractures of the growth plate. AJR 155: 1261-1265
4. Petit P, Panuel M, Faure F, et al (1996) Acute fracture of the distal tibial physis: role of gradient-echo MR imaging verses plain film examination. AJR 166: 1203-1206 
5. White PG, Mah JY, Friedman L (1994) Magnetic resonance imaging in acute physeal injuries. Skeletal Radiol 23: 627-631

6. Mink JG, Deutsch AL (1989) Occult cartilage and bone injuries of the knee: detection, classification, and assessment with MR imaging. Radiology 170: 823-829

7. Berger JE, Ofstein RA, Jackson DW, et al (1989) MR demonstration of radiographically occult fractures: what have we been missing? Radiographics 9: $407-436$
8. Kaplan PA, Walker CW, Kilcoyne RF, et al (1992) Occult fracture patterns of the knee associated with anterior cruciate ligament tears: assessment with MR imaging. Radiology 183: 835-838

9. Zobel MS, Borrello JA, Siegel MJ, et al (1994) Pediatric knee MR imaging: pattern of injuries in the immature skeleton. Radiology 190: 397-401

10. King SJ, Carty HML, Brady O (1996) Magnetic resonance imaging of knee injuries in children. Pediatr Radiol 26: 287-290
11. Salter RB, Harris WR (1963) Injuries involving the epiphyseal plate. J Bone Joint Surg Am 45: 587-622

12. Bertin KC, Goble EM (1983) Ligament injuries associated with physeal fractures about the knee. Clin Orthop 177: 188-195

13. Ogden JA (1982) Injury to the growth mechanisms. In: Ogden JA (ed) Skeletal injury in the child. Lea and Febiger, Philadelphia, pp 59-110 